Genes and Genomes (KEGG) pathway analysis proved that the core targets were significantly enriched in bladder cancer, pathways in cancer, and the like. The docking results showed that beta-sitosterol, glycitein, and quercetin had a good binding activity to CRC putative targets.

Conclusions Our work successfully predicted the active ingredients and potential targets of lycium barbarum for CRC and helped to illustrate the pathways and mechanisms of action on a comprehensive level.

\section{IDDF2020-ABS-0037 EXPRESSION AND ENRICHMENT ANALYSIS OF HOXC8 IN ESOPHAGEAL CANCER}

${ }^{1}$ Mingxin Zhang*, ${ }^{2}$ Lingmin Zhang, 'Manli Cui, ${ }^{1}$ Ning Lu, ${ }^{1}$ Jia Wang. ${ }^{1}$ The First Affiliated Hospital of Xi'an Medical University, China; ${ }^{2}$ First Affiliated Hospital, Xi'an Jiaotong University, China

\subsection{6/gutjnl-2020-IDDF.18}

Background To investigate the expression of HOXC8 in esophageal cancer and its possible tumor pathway, in order to provide a reference for future research directions.

Methods The mRNA-Seq data and clinical prognosis data of esophageal cancer dataset were downloaded and preprocessed from the TCGA database. The differential genes were analyzed, and the volcano map and heat map were drawn. The differentially expressed genes were screened visually. The median HOXC8 expression was divided into high expression group and low expression group, and survival analysis was performed using SPSS software. The HOXC8 samples divided into high expression group and low expression group were then subjected to enrichment analysis using GSEA 4.0.1 software, and graphical analysis of multi-GSEA enrichment analysis was performed at the same time.

Results After differential expression analysis of mRNA expression data of 161 esophageal cancer tissues and 11 paracancerous tissues, 3454 differential genes were screened, including 2317 up-regulated genes and 1137 down-regulated genes, and volcano maps were drawn (figure 1). The results of cluster analysis showed that it could effectively distinguish esophageal cancer from adjacent tissues, indicating that the above differential expression results had good accuracy (figure 2). Difference analysis and paired difference analysis showed that HOXC8 was highly expressed in esophageal cancer, and the difference was statistically significant $(\mathrm{P}<0.05)$. Using the median expression of HOXC8 as a boundary, patients were divided into HOXC8 high expression group and HOXC8 low

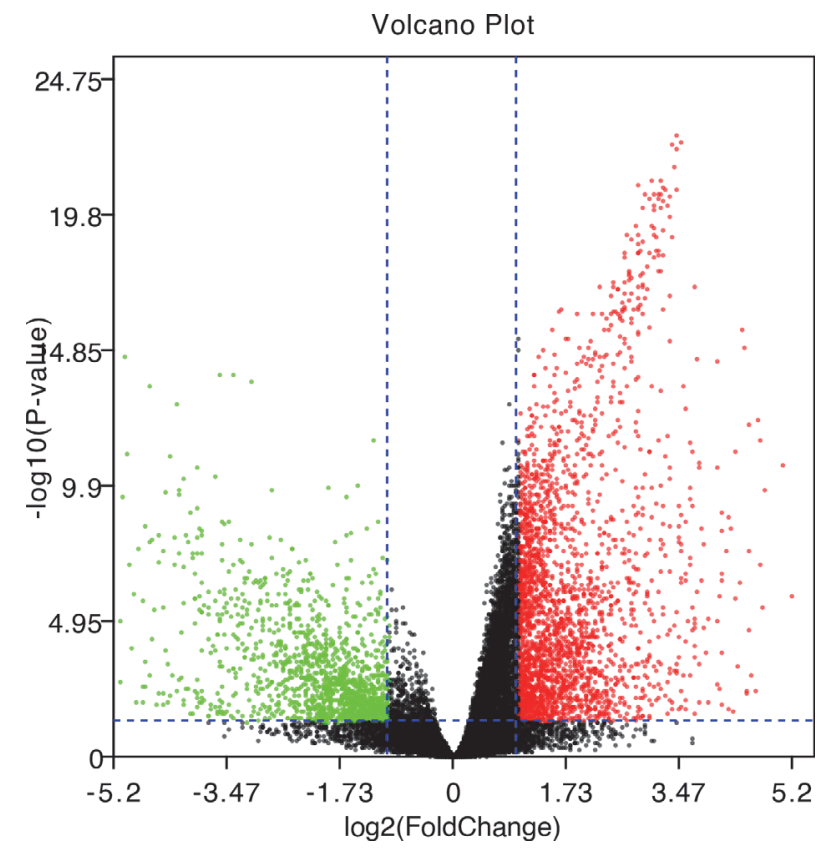

Abstract IDDF2020-ABS-0037 Figure 1

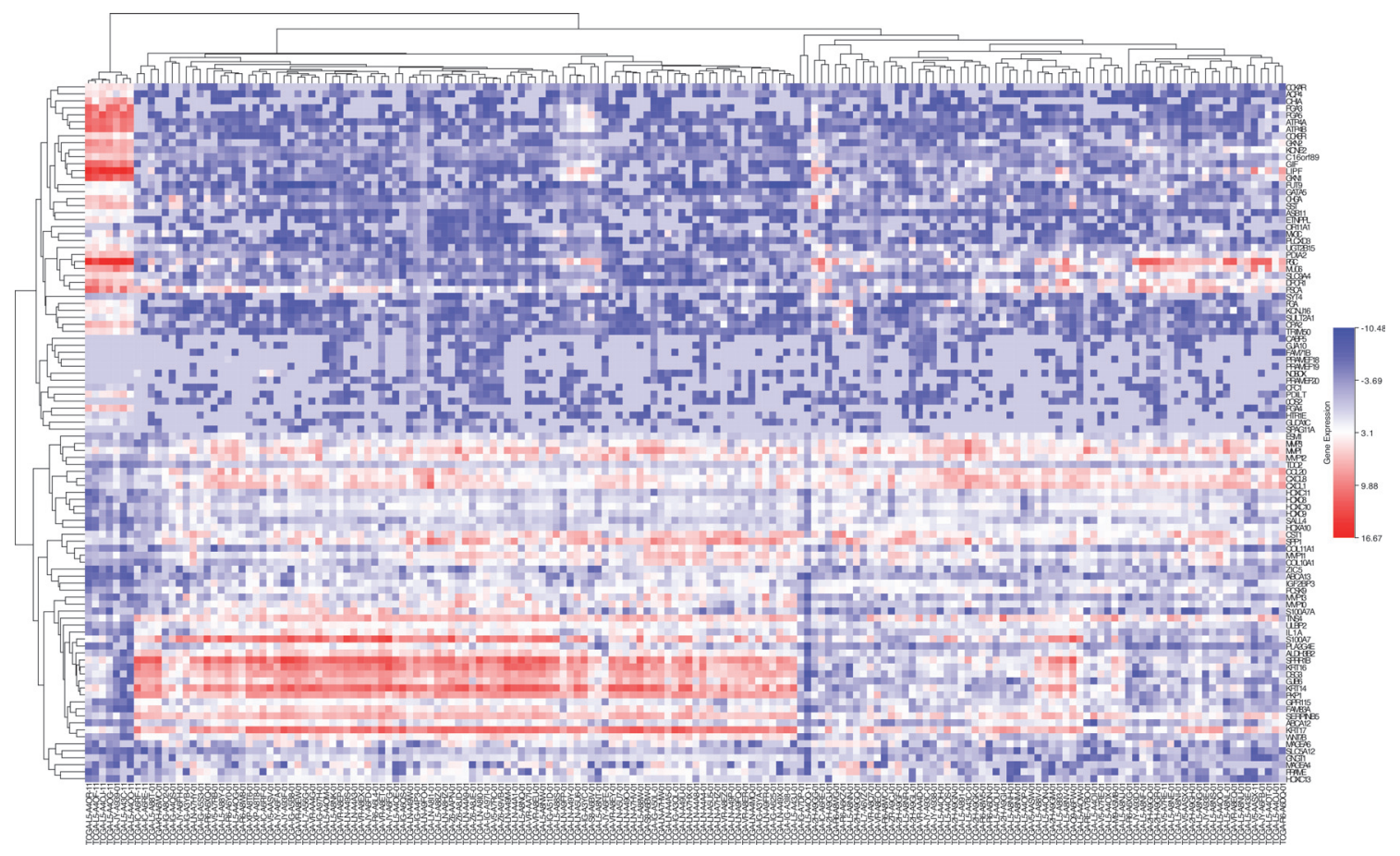

Abstract IDDF2020-ABS-0037 Figure 2 
expression group (figure 3,4). The results showed that patients with high HOXC8 expression had a worse prognosis, suggesting that HOXC8 is an oncogene (figure 5). The results of GSEA analysis showed that high HOXC8 expression was

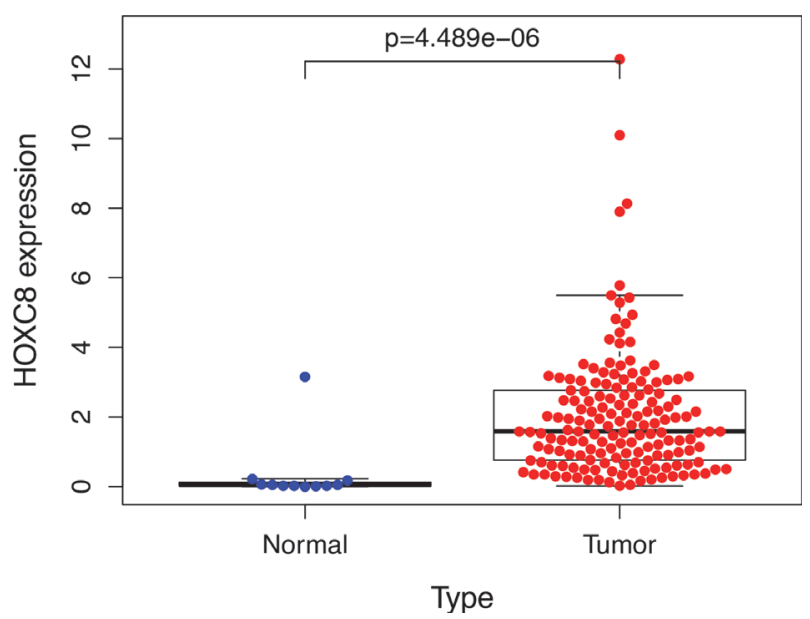

Abstract IDDF2020-ABS-0037 Figure 3

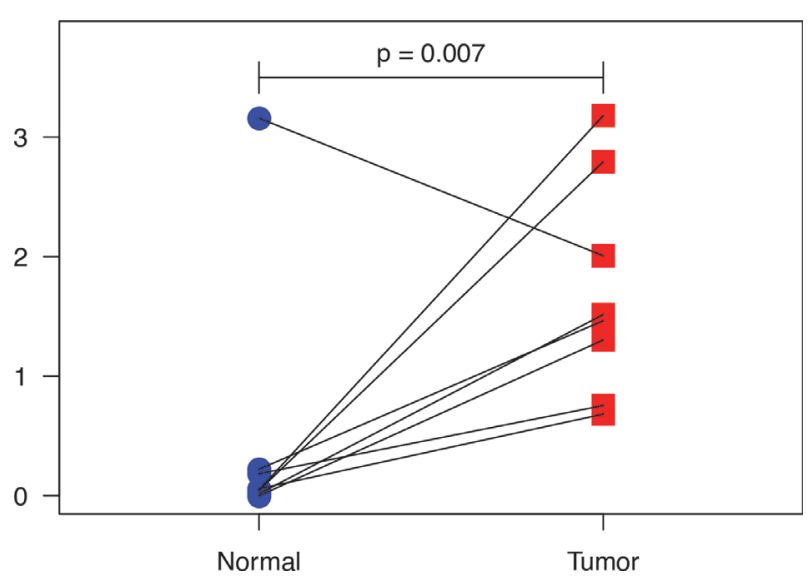

Abstract IDDF2020-ABS-0037 Figure 4

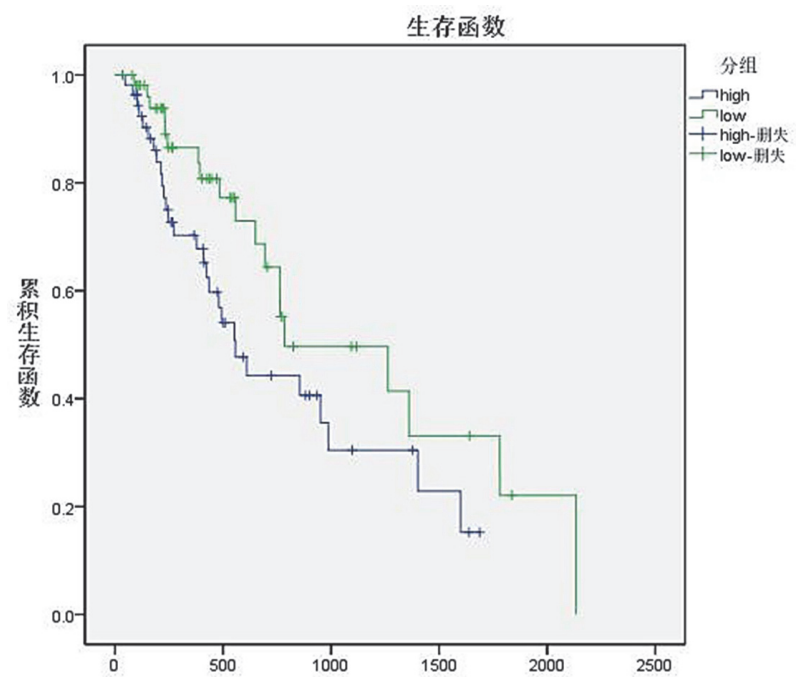

Abstract IDDF2020-ABS-0037 Figure 5

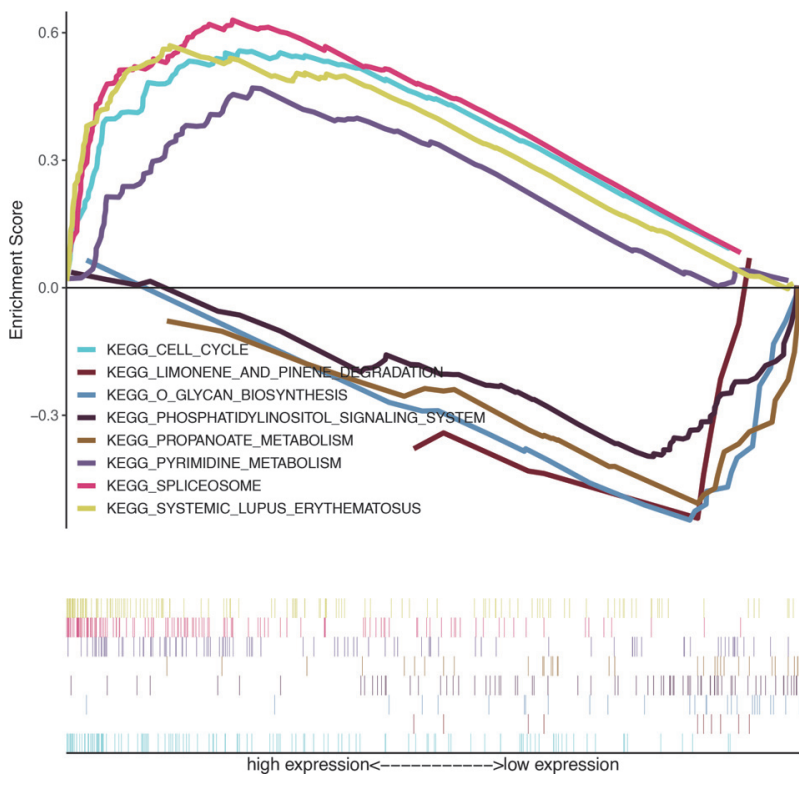

Abstract IDDF2020-ABS-0037 Figure 6

enriched in the cell cycle, splicing and other related gene sets, while low HOXC8 expression was enriched in phosphatidylinositol signaling pathway and other related gene sets (figure 6).

Conclusions HOXC8 may be one of the biomarkers for early diagnosis and prognosis of esophageal cancer. This will further study the role of HOXC8 in esophageal cancer in the future. The mechanism provides the basis and direction.

\section{IDDF2020-ABS-0046 HOXC6 PROMOTES METASTASIS BY RECRUITING MACROPHAGES AND ORCHESTRATING THE IL6/HOXC6/DKK1/ WNT/B-CATENIN AXIS IN RIGHT-SIDED COLON CANCER}

Lina Qi* Biting Zhou, Jiani Chen, Wangxiong Hu. Zhejiang University, China

\subsection{6/gutjnl-2020-IDDF.19}

Background Patients with right-sided colon cancer (RCC) generally have a poorer prognosis than those with left-sided colon cancer (LCC). We previously found that homeobox C6 (HOXC6) was the most significantly upregulated gene in RCC compared to LCC. However, it remains unclear whether HOXC6 plays a role in tumor proliferation and metastasis. Our study aimed to explore the potential oncogenic role and the detailed molecular mechanism of HOXC6 in RCC.

Methods Expression datasets from The Cancer Genome Atlas (TCGA) and Gene Expression Omnibus (GEO) databases were used to uncover the underlying oncogenic role of HOXC6 in RCC. CCK8, transwell, subcutaneous tumor cell injection and tail vein tumor cell injection assays were used to evaluate the proliferation, migration, and invasion of tumor cells in vitro and in vivo. Immunohistochemistry and immunofluorescence were performed to evaluate the correlation of HOXC6 and M2 macrophage infiltration. Western blot, qRT-PCR, ELISA, co-IP, and luciferase reporter assays were performed to examine the association of HOXC6 with epithelial-mesenchymal transition (EMT). 